\title{
A Quality of service-enabled Pricing Approach for Heterogeneous Wireless Access Networks
}

\author{
Antonio Guerrero-Ibañez ${ }^{\mathrm{a}}$, Carlos Flores-Cortés ${ }^{\mathrm{b}}$ \\ School of Telematics, University of Colima. \\ Av. Universidad \#333, Colonia Las Víboras, 28040 - \\ Colima, México, (+52) 3123161075 , \\ antonio_guerrero@ucol.mx, cflores@ucol.mx.
}

\author{
Antoni Barba ${ }^{\mathrm{c}}$, Angelica Reyes ${ }^{\mathrm{d}}$ \\ ${ }^{\mathrm{c}}$ Telematics Engineering Department \\ ${ }^{\mathrm{d}}$ Computer Architecture Department \\ Technical University of Catalonia, Jordi Girona 1-3, Edifici \\ C3, 08028- Barcelona, Spain, (+34) 934016022 \\ telabm@entel.upc.edu, mreyes@ac.upc.edu
}

\begin{abstract}
Heterogeneous wireless access networks (HWAN) will create a market for the delivery of an extensive collection of novel and attractive services and contents. Accounting and pricing the ubiquitous services will play a key role from both service providers and users point of view. By one hand, wireless service providers (WSP) look for the maximum revenue and utilization rate and by other hand users will select service providers depending on the offered price and the Quality of Service (QoS) assurance. In such a market-based scenario, accounting will become an effective tool that satisfies the users' service demands and maximizes the revenue of the providers.
\end{abstract}

For WSP is of vital importance to define a charging and accounting architecture that supports fast and easy the provision of existent and new services with the best quality. In this sense, a dynamical network selection mechanism has to be developed to decide an appropriate radio access cell or technology for a specific service. Additionally, service providers must consider alternative solutions to situations where the best quality for the requested service cannot be provided, allowing them maintaining a high user satisfaction level.

In this paper we propose a simple QoS-based dynamic pricing approach for services provisioning into a heterogeneous wireless access networks environment which attempts to increase user's satisfaction level by firstly, maximizing the provided QoS level, and secondly, by applying dynamic pricing strategies according to the QoS. These strategies will allow service providers to maximize their profits. Simulations results demonstrate that the proposed dynamic pricing approach benefits both users and WSP. Results suggest that users have better overall satisfaction due to a better QoS level and fairer prices. The analysis shows that our proposed pricing approach helps increasing WSPs profits compared to the application of the flat-rate pricing model into a competitive market-model.

Keyword: accounting, pricing, quality of service, user satisfaction, revenue.

\section{INTRODUCTION}

Development in wireless access technologies and sophisticated personal user devices are driving the way towards a heterogeneous wireless access networks (HWAN) environment, which will support a wide range of services having a variety of quality of service (QoS) requirements (such as low latency, high bit rate, low error rate, among others).

Since the wireless service providers' point of view, it will be essential to enable versatile and adaptable pricing strategy models that allow them to reach a high competition level, which can be applied as specific offers (according to user profiles, network priorities and service promotions, among other options) and can support fast and easy provision of existent and new services with the best quality. Additionally, WSPs must consider alternative solutions to situations where the best quality for the requested service cannot be provided, allowing them to maintain a high user satisfaction level.

In this paper we present a QoS-based dynamic pricing approach for service provisioning in a heterogeneous wireless networks environment where requirements of new services demand efficient, and flexible pricing strategies and charging mechanisms. We propose an adaptable scheme to changeable environments, which satisfies demands of the users and allows service providers maximizing their revenue. Our system is called QoSPMS (Quality of Service Pricing Management System). We operate with the existing accounting protocols, keeping compatibility with current developments (Diameter [1], Context Transfer Protocol [2], etc).

In order to maximize user's satisfaction, QoSPMS defines an access network selection mechanism explained later on, that attempts to allocate the most appropriate network for the requested service, satisfying user's demands and optimizing the usage of resources. As a result, service providers may increase their profits too. Additionally, when the QoS requirements cannot be fulfilled, a dynamic pricing strategy and a negotiation procedure are defined.

\section{PROPOSED ACCOUNTING MANAGEMENT APPROACH}

The evolution of pricing strategies for telecommunication mobile services is in a continuous process. Several pricing strategies have been proposed and some of them have been implemented in the commercial environment. However, pricing in heterogeneous wireless access networks is still a challenge that requires more research.

Regarding control strategies several solutions have been 
proposed in the literature. In [3], a pricing policy for multiple competing ISPs (Internet Service Providers) using a threat strategy is presented. Reference [4] shows a proposal in which authors use game theory to analyze the impact in the cost based on the economic interests of a wireless access point owner and his/her paying client. In reference [5] several problems for resource allocation and base-station assignment in CDMA (Code division multiple access) networks are studied. Other projects focus on evaluating the revenue maximization and pricing problems [6-7].

Unlike other pricing management proposals, which reflect the maximization of profits from the points of view either of service provider or users, our proposal considers both (service providers and users' satisfaction) by using a flexible QoSbased pricing approach for heterogeneous wireless access networks.

We focus on the pricing management by proposing the definition of algorithms and strategies that allow keeping an acceptable user's satisfaction level and maximizing the obtained benefits for both users and service providers. In the rest of the section we discuss in detail our dynamic pricing strategy approach for heterogeneous wireless access networks.

\section{A. Management control policies}

QoSPMS is based on the IETF policy model [8]. When new network resources become active, policies can change and the business needs and models can vary accordingly. These changes are actuated through high-level policies that are translated into low-level localized actions allowing achievement of overall system goals.

Our approach specifies three policy classes: access policies, negotiation policies, and pricing and charging policies. The main objective of the policies is satisfying user's service demands by firstly, maximizing the provided QoS level; and secondly, when the QoS level cannot be provided, by applying policies with fairer tariffs attempting to recover the user's satisfaction level as soon as possible.

Access policies allow managing the allocation procedure of the service requests. When a request arrives, the access policy executes a preliminary admission. The idea is to maximize the QoS level provided to the user. When the network can provide the required QoS, the access policy triggers the selection mechanism in order to select the best cell for the service. Otherwise, the access policy triggers the negotiation policy.

Negotiation policies can be used for new QoS level allocation. These policies define a negotiation model which is used when the QoS level cannot be provided. Some considerations are analyzed during the negotiation process. Since dropping an ongoing request is more serious than blocking a new one, the negotiation policy treats handoff requests differently. This measure is related with the user perception level about service continuity.

Each service provider has its own tariffs for the service. However, tariffs should consider a dynamic model according to current network conditions. In our case, pricing policies help adjusting in a dynamic way the price for the service within the changeable behavior of the environment.

\section{B. Access network selection algorithm}

Currently, there is no automated way for the user terminal to intelligently select the most appropriate network from a heterogeneous mix of wireless and mobile access technologies, for accessing the desired service. There are works which have been focused to solve the problem from the service providers' point of view, as shown in the work [9] where the main objective is to maximize the overall network capacity. However they do not consider user's preferences in the decision. In [10] authors propose a mechanism of access network selection from user's perspective which is focused in handling the jitters incurred by transmission on multiple interfaces. But they do not consider a differentiation among the several kind of services and their specific requirements.

The purpose of our selection algorithm is to get the most efficient and suitable access network, by dynamically placing individual connections in the best available networks at any location within a complex network environment, meeting the service's QoS requirements and optimizing the usage of resources. The algorithm is called QoS-based Network Selection Algorithm (QNSA), and as we will see it considers user's preferences adding intelligence to the selection procedure through the connection records, also the algorithm considers network capabilities (e.g., bandwidth), current network conditions and service requirements.

The QNSA is based on the definition of a utility function and the use of a user connection record, named UCP (User Connection Profile). Network selection algorithm is designed to guide user's behavior in cooperative way. QNSA defines two essential mechanisms for selecting the best access network for the service: the utility function, and the user connection profile (UCP). The following sections describe in details the defined mechanisms.

\section{1) Utility function}

The utility function (ec-1) defines a set of parameters which are evaluated in order to select the most suitable access network for the desired service. Parameters are divided into categories: service parameters, network parameters, and user's preferences.

Category of services parameters refers to information of service requirements. Category of network parameters is related to the current network conditions. Finally, user's preferences represent relevant values for some factors as price and QoS level. Values of these parameters are defined as a weight. Users may specify the importance or weights of each parameter which sum to 1 .

Each service has its own requirements. We consider the service classes defined by the $3^{\text {rd }}$ Generation Partnership 
Project (3GPP) [11]. 3GPP defines four service classes: conversational class, streaming class, interactive class, and background class. The main distinguishing factor between these QoS classes is how delay sensitive the traffic is: conversational class is meant for traffic which is very delay sensitive while background class is the most delay insensitive traffic class.

Conversational and Streaming classes are mainly intended to be used to carry real-time traffic flows.

Parameters used in the utility function are bitrate (b), delay (d), jitter (j), error rate (e), and cost or price (c). However, the concept can be extended to include other QoS parameters. In this way, we define the utility function for the access network $k$ for the service $s$ as follow:

$$
\left.U_{k}(s)=p_{\text {price }} \times f_{k, s}(c)+p_{Q o s} \downarrow f_{k, s}(b) \times f_{k, s}(d) \times f_{k, s}(j) \times f_{k, s}(e)\right\rfloor \quad(e c-1)
$$

Where $f_{k, s}(x)$, for $x=c, b, d, j$, e represents the evaluation functions for bitrate, delay, jitter, error rate, and cost (ec-2). The function for each parameter defines the difference between the estimated value for the specific service $\left(X_{\text {defined }}\right)$ and the current value provided by the network $\left(X_{\text {current }}\right)$. $P_{\text {price }}$ and $P_{Q o S}$ represent the user's preferences for price and quality of service respectively.

Some considerations are taken in count when the evaluation function is calculated for each parameter. For bitrate, we are interested that the network provides the best bitrate for the service, however, other parameters like delay, jitter, and error rate need that the network provides the minor possible value.

In this way, we consider $f_{k, s}(x)$ as follow:

Bitrate

$$
f_{k, s}(x)=\left\{\begin{array}{l}
0, \quad x_{\text {current }}<x_{\text {defined }} \\
f\left(x_{\text {current }}, x_{\text {defined }}\right)
\end{array}\right.
$$

Delay, jitter, error rate, price

$$
f_{k, s}(x)=\left\{\begin{array}{l}
0, \quad x_{\text {current }}>x_{\text {defined }} \\
f\left(x_{\text {current }}, x_{\text {defined }}\right)
\end{array}\right.
$$

Evaluation function $f\left(x_{\text {current }}, x_{\text {defined }}\right)$ defines two possible conditions for the evaluation of each parameter. A value of zero if the network cannot provide the QoS level, otherwise, the evaluation function gets a value from the current and defined values. This first evaluation allows reducing the set of candidate networks.

$$
f\left(x_{\text {current }}, x_{\text {defined }}\right)=\exp \left[-\frac{\left(x_{\text {current }}-x_{\text {defined }}\right)^{2}}{\left(2 x_{\text {defined }}^{2}\right)}\right]+F_{g}
$$

Where $F_{g}$ represents the gain factor for the evaluated parameter (ec-3).
Bitrate

$$
F_{g}=\left(x_{\text {current }}-x_{\text {defined }}\right) / 100 *\left(2 x_{\text {defined }}^{2}\right)
$$

Delay, jitter, error rate, price

$$
F_{g}=\left(x_{\text {defined }}-x_{\text {current }}\right) / 100 *\left(2 x_{\text {defined }}^{2}\right)
$$

Final value of the utility function will be in an interval from 0 to 1 . If the QoS level that the network can provide is almost the same that the required level, the utility function will be almost 1 . In this way, the selected access network will be determined by:

$$
\text { Selected_network }(s)=\max \left(U_{k}(s)\right)
$$

\section{2) User connection profile}

This component is based on the idea that, if the user has a regular pattern, the locations and services that the user accesses could be recorded. These records will give a certain intelligence level which can be used in the selection process, so we could avoid or reduce the time processing that the equipment has to spend in calculations during the selection activity.

The learning model we propose is based on a track record, named User Connection Profile (UCP), which stores all the information about the selection decisions that have been made when the user accessed to services.

However, early decisions of the selection algorithm are taken based on the utility function. When we have several records about connection for that service, the decision is made based on this profile, and if the selected network does not have the required resources for the service the algorithm applies the utility function.

\section{Dynamic pricing strategy}

The evolution of pricing and charging schemes for telecommunications and Internet services is a continuous process. A few pricing schemes are widely used in the Internet and mobile environments today [12]: access-rate-dependent charge, volume-dependent charge, or a combination of the both. In static pricing strategies, the price of the different services is either fixed or is only changed at specific period of the day.

Dynamic pricing considers the price as an additional network parameter that can be changed during relatively short periods of time in order that the network operates always with the optimum price according to the available resources and the existing demand.

Our model considers both static and dynamic pricing strategies in order to keep the user's satisfaction level. So, we 
define a QoS-enabled dynamic pricing strategy that considers a simple method for the definition of the adequate tariff for the service. Using both strategies, our model allows WSPs to define static tariffs for specific periods of time, and at the same time, applying dynamic tariffs that adjust the static tariff according to the assigned QoS level, see figure 2. The model is based on the definition of subjective categories that represent QoS tolerance levels. These categories are included within a profile that we named QoS Satisfaction profile (QSP).

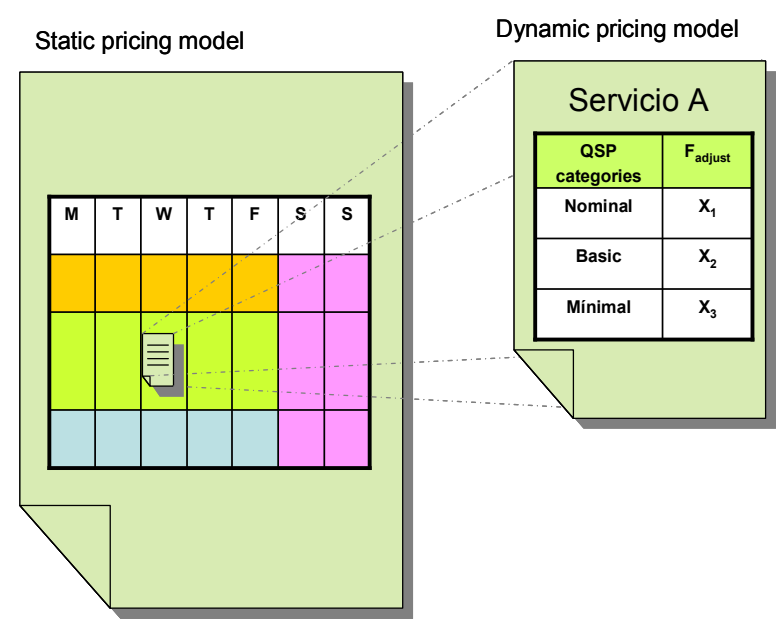

Figure 2. Representation of the QoSPAMS dynamic pricing strategy.

\section{1) Quality of service satisfaction profile}

QoS satisfaction profile (QSP) is a subjective profile defined by WSPs. QSP is based on the Service Level Agreement (SLA). Within the SLA, the service provider defines a nominal tariff for the service. Additionally, the service provider specifies a minimal tariff in order to protect its profits.

One QSP is defined by each service that a WSP offers to end-users. Each category defined on QSP represents a QoS satisfaction grade. For example, for a service $k$, service providers could create three categories: nominal, basic, and minimal, see figure 3 . The number of categories can vary depending of the service.

Categories represent different Service Level Specifications (SLS) which are generated from SLA. SLS parameters simplify the definition of agreements due to SLS describes service without ambiguity. Categories allow WSPs to define dynamic tariffs that adjust according to QoS level assigned to users. These adjusts should be controlled by flexible mechanisms like control policies.

\section{2) Tariff adjustment procedure}

The procedure defines the price that should be charged for the service according to the WSP's strategy. Three situations are analyzed within the tariff adjustment procedure. First situation considers a current network environment where service provider can provide the QoS level defined by the nominal category. In this case, the price for the service is the nominal tariff.

$$
T_{\text {service }}=T_{\text {nominal }}
$$

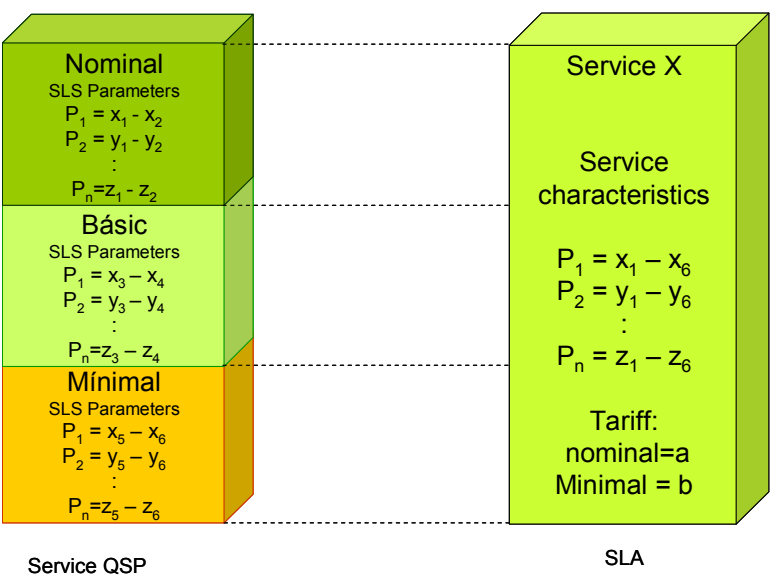

Figure 3. Representation of the Quality of Service Satisfaction Profile.

Second situation represents a current network environment where service provider cannot provide the appropriate QoS level. Tariff is dynamically changed based on the provided QoS level. This tariff could be calculated by ec-3. The nominal tariff is affected by a decrement of price according to the QoS level.

$$
T=T_{\text {no } \min a l} \times F_{\text {adjust }} \quad(e c-3)
$$

The factor that updates the nominal price is named adjustment factor $\left(F_{\text {adjust }}\right)$. This factor represents the discount applied to the price for the degradation of the quality of service.

Finally, the last situation defines a current network environment where the service provider can provide a level less than the minimum specified. In this situation, the provider could define any model where would apply the minimal tariff or in the worst case scenario a free access while the service provider recover the optimum QoS level. In this way, the tariff could be defined as follow:

$$
T=T_{\text {minimal }}
$$

\section{EVALUATION AND RESULTS}

In order to evaluate the performance of QoSPMS, we carry out some simulations in a discrete-event simulator developed in $\mathrm{C}++[13]$. QoSPMS was evaluated within a detailed scenario with Wi-Fi and $3 \mathrm{G}$ access technologies. The access networks are controlled by two different WSP.

We evaluated the performance of the proposed dynamic pricing strategy in order to analyze the revenues that WSP can get applying the proposed strategy. Moreover we examined the performance of the pricing strategy in comparison with 
other models like pricing based on flat-rate. Finally, we compared the impact of each pricing strategy in the user's satisfaction level. We begin by describing how access networks and traffic flows are modeled.

\section{A. Description of the simulated scenario}

We simulate a network composed by four access networks. Each access network represents a management domain. These access networks allow us to represent handover and new connection situations.

WSPs apply different pricing strategies. First WSP defines a flat-rate strategy where user pays a fixed price independently of the QoS level. Second service provider applies the QoSPMS dynamic pricing strategy. We define two variations of the QoSPMS pricing strategy in order to analyze the performance of our approach. Both strategies use a QSP with three categories. However, different discounts are applied in each category. Nominal tariff is the same for both service providers.

In the simulation model, users generated two types of requests, handover and new connection ones. We defined the percentage of handover and new connection considering the distribution of the type of requests based on the study performed about user mobility in urban zones of Barcelona [14]. General studies shown that the transportation media most used in big cities is the vehicle, with values near $40 \%$, whereas the public transportation and pedestrian users have a similar weight of $30 \%$. Nevertheless, a different situation occurs in Barcelona. Study demonstrated that vehicles are used only by $20 \%$ of population as the transportation media. The others transportation media continues being balanced about $40 \%$.

We considered the pedestrian mobility as the percentage of new connection requests due to the almost null handover probability during the average duration of the service. On the other hand, we considered the percentage of users moving in vehicle $(20 \%)$ and in public transportation $(40 \%)$ as the percentage of handover requests in the scenario of simulation $(60 \%)$.

We defined two real-time services (VoIP and videotelephony) and two non real-time services (FTP and web browsing). Service requests were generated using a burst process. We varied the arrival rate from 10 to 30 requests per burst.

\section{B. Obtained Results}

Figure 4 shows the average revenue obtained by each pricing strategy within the scenario defined. As can be noted, the WSP that applied the dynamic pricing strategy obtained the best results in comparison to the WSP that applied flat-rate strategy. In the beginning of the network operation, both strategies obtain similar revenues. This is due to the networks are used equally.
However, as time goes on, the different variants of QoSPMS pricing strategy obtain better revenues and in some cases the increase goes up to $60 \%$ depending on the dynamic strategy applied. On the other hand, the graphic shows that the QoSPMS-1 obtains better results than QoSPMS-2, improving up to $20 \%$ the profits obtained by WSP.

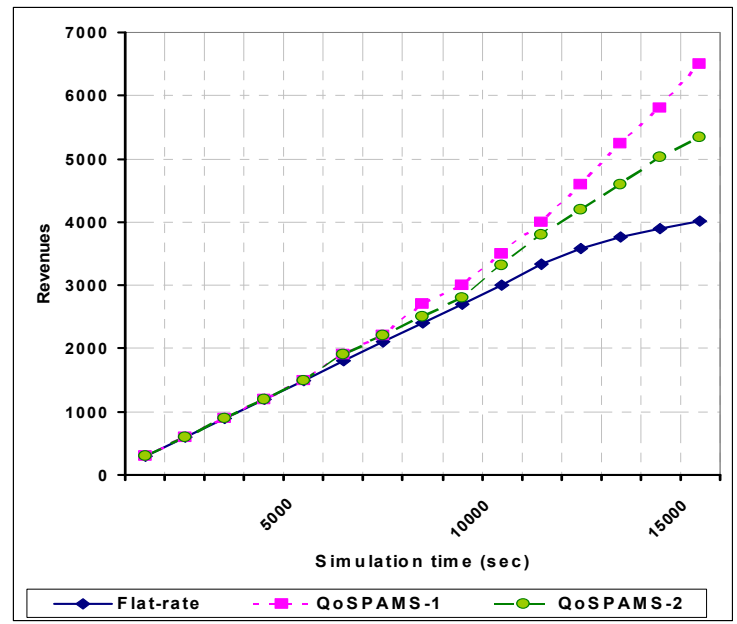

Figure 4. Average revenue obtained by each pricing strategy.

Finally, the results obtained regarding the impact that pricing strategies introduce in the user satisfaction level are presented. The user satisfaction is based on the user costperformance expectation. Figure 5 shows the effects introduced by the pricing strategies.

The results show that strategy based on flat-rate considerably affects the user satisfaction level as the quality of service decreases. QoSPMS strategy reduces the impact of almost $40 \%$ in comparison to flat-rate strategy. Regarding to QoSPMS strategies, they have a similar behavior; however, QoSPMS-1 obtained the best results, improving the user satisfaction level up to $4 \%$ in comparison to QoSPAMS-2 when the QoS level is reduced to the minimum level.

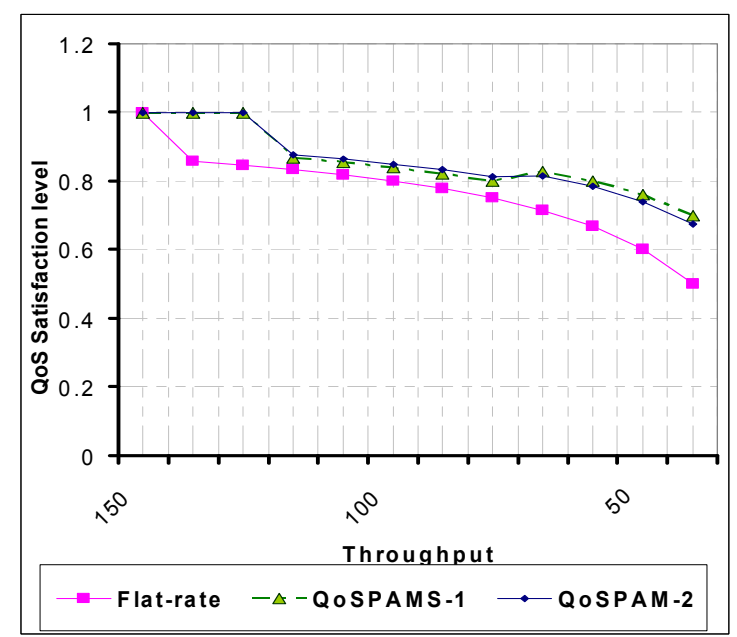

Figure 5. effects of pricing strategies in the user's satisfaction level. 


\section{Conclusions}

Technological developments and the vision towards integration of emerging and existing technologies suggest the evolution toward a heterogeneous wireless access networks environment attempting to give solution to the demands of the modern society. Among the demands, we can stand out satisfying a high mobility of the users and the seamless access, without restrictions neither in the coverage area, the access method, or the instant of time.

In this paper, we presented an approach based on dynamic pricing strategies that formulates the interaction between providers and users in a market-based environment. This solution, named QoSPMS, is applied into a multi-domain, multi-WSP network environment. QoSPMS considers the satisfaction of both wireless service providers and users, valuing that, a user satisfied with a service is willing to use it in the future and to recommend the service to other users, producing an increase of profits for the service provider.

To achieve this, we defined an access network selection mechanism that attempts to choose the appropriate network for each requested service, maximizing the provided QoS level. As a second measure, when the suitable QoS cannot be fulfilled, we define a dynamic pricing strategy that allows service providers to adapt the price according to the supplied QoS level. Pricing strategy is based on subjective categories that represent QoS tolerance levels.

We proved by simulations that our proposed pricing approach helps both users and service providers to increase their profits. With the help of simulation, we demonstrate how the proposed dynamic pricing mechanism and QoS-enabled network selection strategy achieved better performance for both service providers and users. This efficiency is based on the provision of quality of services and the adjustment of tariffs applied to the services, improving the user's satisfaction, resulting in an increase of revenues for service providers.

\section{ACKNOWLEDGMENTS}

This work was partially supported by PROMEP under grant No. promep/103.5/08/4113 provided to the first author and by the Spanish MEC project P2PGrid TIN2007-68050-C03.

\section{REFERENCES}

[1] 3GPP TR 32.299 v 8.0.0. Telecommunication management; charging management; Diameter charging applications (Release 8). September 2007.

[2] J. Loughney, Ed., M. Nakhjiri, C. Perkins, R. Koodli. Context Transfer Protocol (CXTP). IETF Request for comments RFC 4067, July 2005.
[3] S. Shakkottai, R. Srikant, Economics of network pricing with multiple ISPs, in: Proc. of IEEE INFOCOM, Vol. 1, 2005, pp. 184_194.

[4] J. Musacchio, J. Walrand, WiFi access point pricing as a dynamic game, IEEE/ACM Transactions on Networking 14(2), 289-301. 2006.

[5] S.C.M. Lee, J.W.J. Jiang, J.C.S. Lui, Chiu Dah-Ming, Interplay of ISPs: Distributed resource allocation and revenue maximization, in: 26th IEEE International Conference on Distributed Computing Systems, 2006.

[6] M. Mandjes, Pricing strategies under heterogeneous service requirements, Proceedings of IEEE INFOCOM, 2003, Vol. 2 pp.1210_1220.

[7] Xin Wang, H. Schulzrinne, Pricing network resources for adaptive applications, IEEE/ACM Transactions on Networking 14 (3) (2006) 506_519.

[8] A. Westerinen, J. Schnizlein, J. Strassner, M. Scherling, B. Quinn, S. Herzog, A. Huynh, M. Carlson. J. Perry, S. Waldbusser. Terminology for Policy-Based Management. IETF Request for Comments RFC 3198, November 2001.

[9] G. Fodor, A. Furuskar, and J. Lundsjo. On access selection techniques in always best connected networks, in ITC Specialist Seminar on Performance Evaluation of Wireless and Mobile Systems, August 2004.

[10] K. Chebrolu and R. Rao. Communication using multiple wireless interfaces. in Proceedings of the IEEE WCNC, March 2002.

[11] 3GPP TS 23.107 v 6.4.0. Quality of Service (QoS) concept and architecture (Release 6). March 2006.

[12] L. Badia, M. Lindström, J. Zander, M. Zorzi. Demand and Pricing effects on the radio resource allocation of multimedia communication services. IEEE Global Telecommunications Conference Globecomm '03, vol. 7, pp. 4116-4121, December 2003.

[13] Omnet ++ Discrete Event Simulation System, http://www.omnetpp.org/.

[14] M. Berjeno, F. Magrynià. Comparación de tiempos de trayectos metro-a pie-bici en la zona urbana de Barcelona, Tesina, Technical University of Catalonia, January 2007. 